\title{
Lossless Image Compression Using an Edge Adapted Lifting Predictor
}

\author{
Ömer N. Gerek* \\ Anadolu University, \\ Department of Electrical and Electronics Eng., \\ Eskişehir TR-26470, Turkey, \\ E-mail: ongerek@anadolu.edu.tr
}

\section{INTRODUCTION}

Lifting implementations of particular wavelets have received a wide range of interest in various image coding applications. Although practically all of the filter-bank style wavelet implementations can be converted to the lifting style implementation (Figure 1), the conceptually interesting portion of the work corresponds to the, so called, prediction part $(P(z))$ of the lifting stage[1]. In this part, the signal is split into polyphase components, and one of the polyphase domains is used to predict the values belonging to the other polyphase component. By designing a successful prediction filter, one tries to minimize the signal energy of the lower branch, $y_{1}[n]$. During this prediction design, nonlinear filters [2] as well as signal adapted filters [3, 4, 5] were reported in the literature. The practical utilizations of the lifting strategy includes compression and analysis of images and video $[6,7]$.

In case of image and/or video processing, the prediction filter design idea can be extended to two or more dimensions. In [8], [9], and [10], such 2-D extensions of the lifting structures were examined, which fundamentally resembles the idea of this work.

As an illustration in the 2-D case, consider a polyphase decomposition of an image in the horizontal direction as

*O. N. Gerek's work is supported by Anadolu University Research Fund under Contract No. 030263.

${ }^{\dagger}$ A. E. Cetin's work is partially funded by TUBITAK and TUBA (Turkish Academy of Sciences) GEBIP Programme. \begin{abstract}
regions with diagonal gradient. We have obtained good compresregions with diagonal gradient. We have obtained good compres-
sion results with conventional lossless wavelet coders.

Abstract- We present a novel and computationally simple pre-
iction stage in a Daubechies 5/3 - like lifting structure for lossless image compression. In the $5 / 3$ wavelet, the prediction filter predicts the value of an odd-indexed polyphase component as the
mean of its immediate neighbors belonging to the even-indexed polyphase components. The new edge adaptive predictor, however, predicts according to a local gradient direction estimator of the image. As a result, the prediction domain is allowed to flip +
\end{abstract}

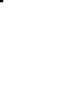

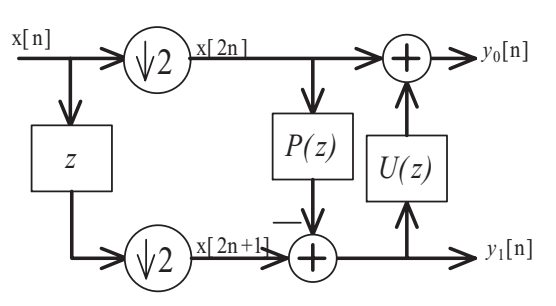

Fig. 1. Lifting analysis stage.

\author{
A. Enis Çetin ${ }^{\dagger}$ \\ Bilkent University, \\ Department of Electrical and Electronics Eng., \\ Bilkent, Ankara TR-06533, Turkey \\ E-mail: cetin@bilkent.edu.tr
}

. 1. Lifting analysis stage.

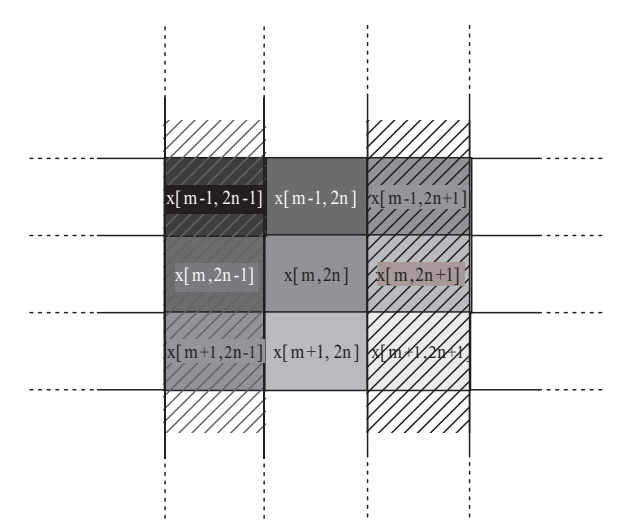

Fig. 2. A sample image segment with south-east gradient.

shown in Figure 2. In this figure, the dashed columns constitute a prediction domain for the pixels in columns that are not dashed. Classically, the separable process considers only one row of such an image data, and considers the prediction problem in 1-D. In this case, the low-pass and highpass subband filters are obtained by the equations $H_{i}(z)=$ $H_{i, e v}\left(z^{2}\right)+z^{-1} H_{i, o d d}\left(z^{2}\right)$, for $i=0,1$, where $i=0$ corresponds to the low-pass, $i=1$ corresponds to the high-pass filter, and the even and odd filters $H_{i, e v}(z)$ and $H_{i, e v}(z)$ are described in terms of lifting stage filters as in Eq. 1. This relation is fairly straightforward, therefore an efficiently designed $P(z)$ automatically corresponds to a successful subband filter-bank.

$$
\left[\begin{array}{cc}
H_{0, e v}(z) & H_{0, \text { odd }}(z) \\
H_{1, e v}(z) & H_{1, \text { odd }}(z)
\end{array}\right]=\left[\begin{array}{cc}
1-P(z) U(z) & U(z) \\
-P(z) & 1
\end{array}\right]
$$


The Daubechies 5/3 wavelet is a simple and powerful wavelet. It has an efficient set of filter coefficients which consist of simple integer fractions:

$h_{0}=[-1 / 8,1 / 4,3 / 4,1 / 4,-1 / 8]$ and $h_{1}=[-1 / 2,1,-1 / 2]$. Its lifting implementation is even more efficient and can be fastly realized using binary shifting operations:

$$
\begin{aligned}
y_{1}[n]= & x[2 n+1]-(x[2 n]+x[2 n+2]) / 2 \\
y_{0}[n]= & x[2 n]+\left(y_{1}[n-1]+y_{1}[n]\right) / 4 \\
= & -x[2 n-2] / 8+x[2 n-1] / 4 \\
& +3 x[2 n] / 4+x[2 n+1] / 4-x[2 n+2] / 8
\end{aligned}
$$

where $x[n]$ is the input signal, $y_{1}[n]$ is the high-pass detail signal, and $y_{0}[n]$ is the low-pass approximation signal.

It can be noticed that the prediction filter is very short, but, it is an effcient non-causal predictor for a pixel in between two other pixels. Let us consider row-wise processing of an image $x[m, n]$. The prediction filter inherently assumes that the right and left pixels are closely related with the pixels between them. As a result, $(x[m, 2 n-1]+$ $x[m, 2 n+1]) / 2$ will be an accurate estimate of $x[m, 2 n]$. By subtracting this estimate from the true value of $x[m, 2 n]$, a small residue is obtained. This residual signal corresponds to the detail signal obtained by the single stage wavelet transformation. The lifting implementation filter taps of this wavelet are expressed as powers of two leading to a multiplication free realization of the filter-bank [1]. Therefore, although several other linear or nonlinear decomposition structures that are published in the literature report better performance than the $5 / 3$ wavelet using signal adapted filters [1]-[5], the 5/3 wavelet was adopted by the JPEG-2000 image coding standard [11] in its lossless mode.

In this work, we extend the prediction idea of the Daubechies 5/3 lifting implementation into two-dimensions. The motivation behind this extension is that, the vertical or horizontal neighbors of a pixel may not constitute a good prediction domain for a pixel around the portion of an image with diagonal gradient. Let us consider horizontal processing of the image without any loss of generality. In some portions of the image, $x[m, 2 n]$ may be unrelated to $x[m, 2 n-1]$ or $x[m, 2 n+1]$, whereas, some of the four other immediate diagonal neighbors $x[m+1,2 n-1], x[m+1,2 n+1]$, $x[m-1,2 n-1]$ or $x[m-1,2 n+1]$ may be closer to the pixel $x[m, 2 n]$ in value. Therefore, it may be better to use two of these four diagonal neighbors in the prediction stage of the lifting structure in a judicious manner. This corresponds to relaxing the condition that the predictor should use samples from the current row under process. In the next section, we introduce the details of selecting the prediction domain according to the edge gradient direction. We emphasize on the fact that the proposed method is computationally efficient and reversible as long as the approximation domain polyphase samples are kept unchanged. We also introduce an update strategy inside the lifting structure which has a different occurrence sequence as compared to classical lifting structures consisting of predictors followed by updates. Finally, we present lossless image compression results comparing the regular Daubechies 5/3 and 9/7 biorthogonal wavelets with the proposed method.

\section{Edge-Direction Sensitive Prediction}

Our work was motivated by the simplicity of the prediction used in the Daubechies 5/3 wavelet, and the interpolation rule for the missing or dead pixels inside a CCD image sensor. The CCD interpolation algorithm that was proposed in [12] uses a smart way of interpolating a missing CCD pixel value from neighboring pixels. Assume that the sensor corresponding to $x[m, 2 n]$ in Figure 2 is down, therefore we are unable to read the sensor output. The adaptive interpolation algorithm is based on the following principles:

- If the up and down pixel value difference is less than the left and right pixel value difference, then the interpolation value is (up + down $) / 2$, or $x[m, 2 n]=$ $(x[m-1,2 n]+x[m+1,2 n]) / 2$,

- else estimate the missing pixel $x[m, 2 n]$ using its left and right neighbors $($ left + right $) / 2$, or $x[m, 2 n]=$ $(x[m, 2 n-1]+x[m, 2 n+1]) / 2$.

The choice of the interpolation value is based on the fact that, if the horizontal neighboring pixel differences is small, then we do not expect an edge that crosses the local image portion in the horizontal direction. Therefore, the pixel in the center most probably lies in a smoothly varying position between its left and right neighbors. A similar argument can be made for the vertical direction, too. As a result, if there is a vertical (horizontal) edge going through pixel $x[m, 2 n]$ then horizontal (vertical) pixels are used in interpolation. This interpolation strategy gives a good approximation of a possibly missing color sensor output and it improves both the mean-square-error and the subjective quality of the acquired color image in CCD imaging systems.

The problem with this interpolatory prediction is that it does not consider horizontal or vertical polyphase decomposition of the image. For example, if horizontal processing of the image is considered, the odd-indexed columns are not available in the prediction domain. In that particular case, for example, the pixel values $x[m-1,2 n]$ or $x[m+1,2 n]$ are not available for predicting $x[m, 2 n]$. However, the CCD interpolation strategy inspires an idea that $x[m, 2 n-1]$ and $x[m, 2 n+1]$ are not the only possible pixels with which a prediction can be made.

In this work, we allow the use of appropriate polyphase pixels from the rows above and below the pixel of interest for the prediction part of the lifting stage. For example, $x[m, 2 n]$ may also be predicted using $(x[m-1,2 n-1]+x[m+1,2 n+1]) / 2$, which corresponds to the average of the north-west neighbor and the south-east neighbor, or $(x[m+1,2 n-1]+x[m-1,2 n+1]) / 2$, 
which corresponds to the the average of north-east neighbor and the south-west neighbor. As an example, if the local gradient is in the south-east direction, then there is more possibility that the center of the $3 \times 3$ region has a pixel value similar to its north-east and south-west neighbors, which are in a direction orthogonal to the edge gradient. This concept is generalized to the other directions according to the following adaptation rule for the selection of prediction domain pixels. Let us first define: $\Delta_{135}=\mid x[m-1,2 n-$ $1]-x[m+1,2 n+1]\left|, \Delta_{0}=\right| x[m, 2 n-1]-x[m, 2 n+1] \mid$, $\Delta_{45}=|x[m+1,2 n-1]-x[m-1,2 n+1]|$ as absolute differences between the neighbors of the pixel $x[m, 2 n]$.

- If $\Delta_{135}$ is the least among $\Delta_{135}, \Delta_{0}$, and $\Delta_{45}$, then the prediction estimate is given by: $\hat{x}[m, 2 n]=(x[m-1,2 n-1]+x[m+1,2 n+1]) / 2$

- If $\Delta_{0}$ is the least among $\Delta_{135}, \Delta_{0}$, and $\Delta_{45}$, then the prediction estimate is given by: $\hat{x}[m, 2 n]=(x[m, 2 n-1]+x[m, 2 n+1]) / 2$

- If $\Delta_{45}$ is the least among $\Delta_{135}, \Delta_{0}$, and $\Delta_{45}$, then the prediction estimate is given by: $\hat{x}[m, 2 n]=(x[m+1,2 n-1]+x[m-1,2 n+1]) / 2$

In the example shown in Figure 2, the largest gradient is in the south-east direction. As a result, $\Delta_{45}$ is the minimum difference, so the value of $\hat{x}[m, 2 n]$ must be predicted as $(x[m-1,2 n+1]+x[m+1,2 n-1]) / 2$. It must be noted that such a tilted prediction $(P(z))$ does not require transmission of any side information as long as the output subband components are not distorted. In that case, the decoder uses the same directional choice method that was used in encoder, and perfect reconstruction is assured. This prediction rule is computationally simple. The required pixel comparisons and sorting can be implemented by 6 additional subtraction operations. Therefore, the computational efficiency of the Daubechies 5/3 wavelet implementation is kept by introducing no multiplications. It was also reported in [9] that such multi-line lifting realizations can be performed in a memory-efficient manner.

Let us assume horizontal processing, without any loss of generality. A complication that may occur during the described lifting operation is that, since the detail coef£cients contain information from rows above and below, the update flter will feed that information back to the approximation coeffcients. This is an undesirable situation that may be considered as an update leakage. Because of this leakage, the effect of $U(z)$ in the lifting stage deviates from antialiasing low-pass filtering, leading to distortions in lowlow subimages across decomposition scales. This problem can be solved by changing the order of the update $U(z)$ and the prediction $P(z)$ stages of Figure 1. With the proper choice of the low-pass filter, the new $U(z)$ can be performed prior to the prediction, and its implementation still requires no multiplications, so the computational efficiency is retained. For this purpose, we consider the simple Lagrangian half-band low-pass filter: $\mathbf{h}_{3}=\{1 / 4,1 / 2,1 / 4\}$. The z-transform of this filter is

$$
H(z)=\left(1+z\left(U\left(z^{2}\right)\right) / 2\right.
$$

where $U(z)=\frac{1}{2} z^{-1}+\frac{1}{2}$. This low-pass filter followed by down sampling can be implemented in a lifting structure due to the relation known as Noble-Identity, where, $U(z)$ can be implemented by bitwise shift-rights.

After this stage, adaptive prediction algorithm described in the previous section can be implemented. Since the lowpass filtering is performed first, the low-low subimages are as good as those obtained by any sub-band decomposition structure using the third-order Lagrange half-band filter.

The overall structure including the low-pass filter is still computationally comparable to the original implementation of the Daubechies 5/3 wavelet in terms of calculations per lifting operation.

\section{EXPERIMENTAL RESULTS AND CONCLUSIONS}

Modification of the prediction domain pixels according to the edge gradient in the lifting stage has a number of practical advantages. We have experimentally observed that, in a typical test image, among all possible three directions, the possibility of the horizontal direction being the best prediction of $x[m, 2 n]$ is $30.1 \%$. This is slightly less than about one-thirds of the possible predictions. As a result, persistently using horizontal prediction loses chances of making better prediction decisions. On the other hand, our directionally sensitive prediction decision rule catches about 52 $\%$ of the best predictions as described above. This improvement reflects to practical compression results, too. We have observed that the detail images obtained by the edge gradient sensitive method exhibits less signal energy at several decomposition levels in general as in Figure 3.

In order to assure perfect reconstruction and possible asymmetries in the encoder/decoder pair, we applied our structure to lossless compression. The compression is based on the image wavelet tree bit-plane coding, similar to the one that is used in JPEG2000 [11]. No particular interest was given to the optimization of the encoder. Instead, we present results comparing the Daubechies 9/7 and Daubechies $5 / 3$ wavelet performance with the method described here using the same lossless coder. However, we have also observed transform entropy and variance reduction. Therefore, similar results are expected with other lossless wavelet coders. A decomposition level of 4 was selected for 8 bit gray-scale images with size $512 \times 512$. The bit-rate values in terms of bits per pixel (bpp) for a set of test images shown in Table I are generated using Daubechies 9/7 wavelet, Daubechies 5/3 wavelet, and our directionally adaptive method using the half-band anti-aliasing update filter. In general, smaller bit-rates are obtained. 


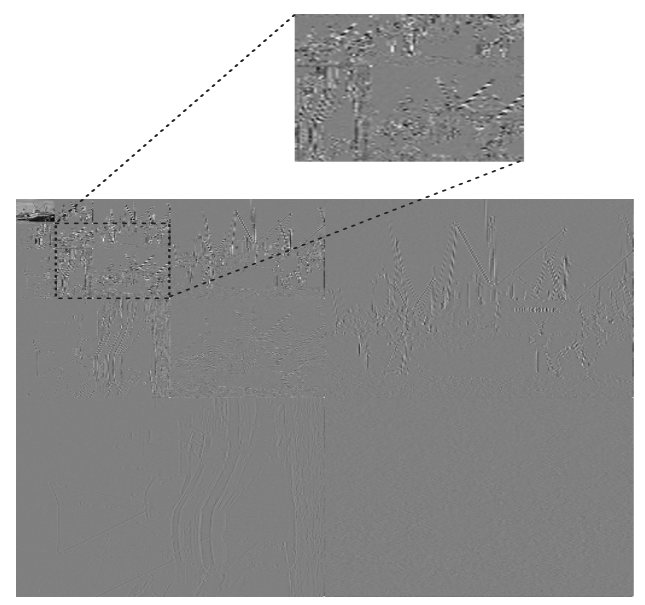

(a)

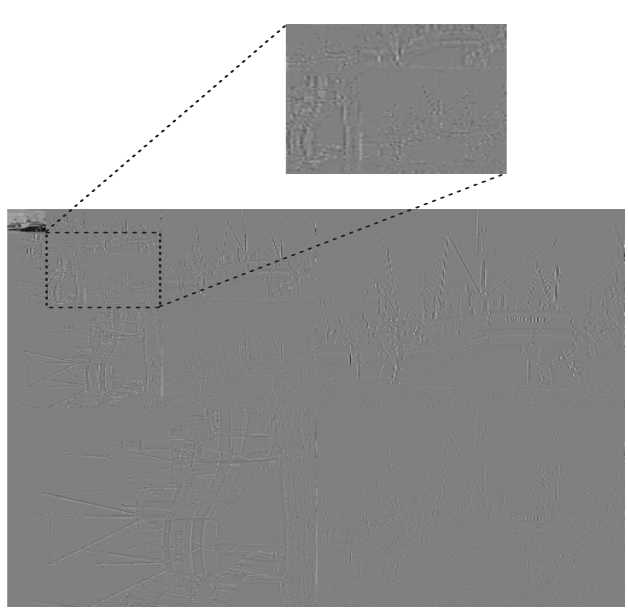

(b)

Fig. 3. Wavelet trees obtained by (a) regular $5 / 3$ wavelet, (b) our method.

\begin{tabular}{|l|c|c|c|}
\hline & Daub. 9/7 & Daub. 5/3 & Our method \\
\hline boats & 4.233 & 4.178 & 4.132 \\
\hline airfield & 5.677 & 5.666 & 5.354 \\
\hline bridge & 5.694 & 5.646 & 5.513 \\
\hline harbor & 4.890 & 4.793 & 4.592 \\
\hline lena & 4.287 & 4.267 & 4.096 \\
\hline barbara & 4.840 & 4.875 & 4.816 \\
\hline houses & 4.851 & 4.791 & 4.635 \\
\hline garden & 4.712 & 4.598 & 4.561 \\
\hline peppers & 4.593 & 4.590 & 4.171 \\
\hline
\end{tabular}

Table 1. Lossless bit-rates for $512 \times 512$ test images.

Although the lossless image compression performances are evaluated here, the edge sensing algorithm was observed to be robust to fine quantization, so the rounding operations in Eq. 2 do not cause problems. We have even obtained reasonable PSNR results with lossy compression at relatively high bit-rates.

The computational complexity of the proposed adaptive filterbank is very low. Our directionally adaptive lifting strategy contains an additional

1. three difference operations to obtain $\Delta_{135}, \Delta_{0}$, and $\Delta_{45}$, and

2. three comparison operations to choose the minimum of $\Delta_{135}, \Delta_{0}$, and $\Delta_{45}$

compared to Daubechies 5/3 wavelet decomposition. The rest of the operations, including the anti-aliasing filtering have identical complexity figures as the original $5 / 3$ lifting implementation. There is neither any integer nor floating point multiplications in the new structure. As a result, our directionally adaptive algorithm keeps the low complexity property of the 5/3 Daubechies wavelet decomposition, and provides good image compression results.

\section{REFERENCES}

[1] I. Daubechies and W. Sweldens, "Factoring Wavelet Transforms into Lifting Steps," Journal of Fourier Analysis and Appl., Vol. 4, Nr. 3, pp. 247-269, 1998.

[2] R. L. Claypoole, G. M. Davis, W. Sweldens W, et al. "Nonlinear wavelet transforms for image coding via lifting,"'IEEE Trans. I.P., Vol.12, No.12, pp.1449-1459, Dec. 2003.

[3] Ö. N. Gerek and A. E. Çetin, "Adaptive Polyphase Subband Decomposition Structures for Image Compression," IEEE Trans. I.P., Vol.9, No.10, pp.1649-1660, Oct. 2000.

[4] G. Piella, B. Pesquet-Popescu, H. Heijmans , "Adaptive update lifting with a decision rule based on derivative filters," IEEE Signal Processing Letters, pp. 329- 332, Oct. 2002.

[5] T. Chan and H. M. Zhou, "Adaptive ENO-Wavelet Transforms for Discontinuous Functions," UCLA Report No. CAM 99-21, June 1999.

[6] G. Pau, C. Tillier, B. Pesquet-Popescu, "Optimization of the Predict Operator in Lifting-Based Motion Compensated Temporal Filtering," SPIE VCIP, San Jose, CA, Jan. 2004.

[7] N. Mehrseresht and D. Taubman, "Adaptively weighted update steps in motion compensated lifting based on scalable video compression," Proc. IEEE Int. Conf. on Image Proc., Vol.2, pp. 771-774, Sep. 2003.

[8] A. Gouze, M. Antonini, M. Barlaud, and B. Mack, "Design of signal-adapted multidimensional lifting scheme for lossy coding," IEEE Trans. I.P., Vol.13, No.12, Dec. 2004.

[9] D. Taubman, "Adaptive, non-separable lifting transforms for image compression," Proc. IEEE Int. Conf. on Image Proc. (ICIP), volume 3, pages 772-776, Oct. 1999.

[10] H. Heijmans, G. Piella, B. Pesquet-Popescu, "Building Adaptive 2D Wavelet Decompositions by Update Lifting", Proc. IEEE Int. Conf. on Image Proc., Rochester, Oct. 2002.

[11] I. J. S. WG01, “JPEG-2000 part-1 standard," ISO/IEC 15444-1.

[12] R. H. Hibbard, "Apparatus and method for adaptively interpolating a full color image utilizing luminance gradients," U.S. Patent 5,382,976. 